\title{
Airline and Aircraft Reliability
}

\author{
Maris Hauka ${ }^{1}$, Jurijs Paramonovs ${ }^{2},{ }^{1-2}$ Institute of Aeronautics, Faculty of Transport and Mechanical Engineering, \\ Riga Technical University
}

\begin{abstract}
Development of the inspection programme of fatigue-prone aircraft construction under limitation of airline fatigue failure rate. The highest economical effectiveness of airline under limitation of fatigue failure rate and failure probability is discussed. For computing is used exponential regression, Monte Carlo method, Log Normal distribution, Markov chains and semi-Markov process theory. The minimax approach is offered for processing the results of full-scale fatigue approval test of an airframe. Fatigue crack parameters and numerical examples are given and explained.
\end{abstract}

Keywords - Inspection programme, Markov chains, minimax, reliability, fatigue crack, exponential approximation.

\section{INTRODUCTION}

The fatigue failure probability (FFP) of fatigue-prone aircraft (AC) and fatigue failure rate (FFR) of airline (AL) are problems of high priority. A lot of papers and books examine these problems and offer possible solutions [1] - [9] where the Markov chains (MC) and semi-Markov process with reward (SMPW) theories [10] - [12] are offered to solve these problems, using exponential approximation of fatigue crack size growth function, (1) where $\alpha, Q$ are parameters of fatigue crack trajectory (PFCT).

$$
a(t)=\alpha \exp (Q t)
$$

The value $\alpha$ is called the equivalent initial flow size (EIFS). (Note, it is not a real initial flow size; it is only a parameter of exponential approximation of fatigue crack trajectory!) The value $Q$ defines the speed of fatigue crack size growth on a logarithmic scale: $\log (a(t))=\log \alpha+Q t$. PFCT are random variables. It is supposed that the cumulative distribution function (cdf) of the vector $(\alpha, Q)$ is known, but a certain parameter of this cdf, $\theta$ is not known. Estimation of $\theta$ and the choice of inspection programme under condition of limitation FFP up to a specified life (AC retirement age), $t_{S L}$, or limitation of FFR of AL can be achieved using minimax processing of results of observation of some random fatigue cracks during $\mathrm{AC}$ type full-scale fatigue approval test. A specific feature of the approval test is a decision to redesign the new AC type if some reliability requirements are not met. In [1], it was assumed that $\alpha$ was some constant. In this paper this assumption is eliminated.

\section{Minimax Choice of InSPECTION Programme}

Despite all the simplicity, formula (1) gives us a rather comprehensible result in the interval $\left(t_{d}, t_{c}\right)$, where $t_{d}$ is a time when the crack becomes detectable [13-15] $\left(a\left(t_{d}\right)=a_{d}\right)$
(2) and $t_{c}$ is a time when the crack reaches its critical size $\left(a\left(t_{c}\right)=a_{c}\right)(3)$ and fatigue failure takes place (see Fig. 1).

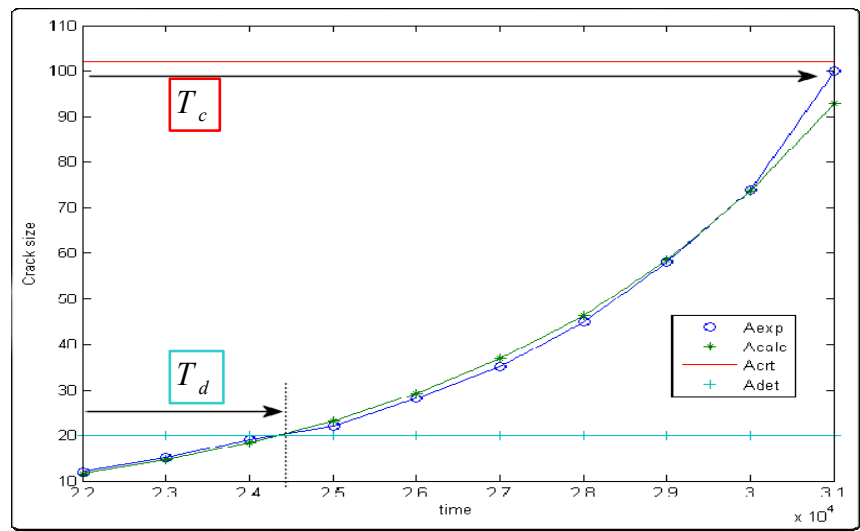

Fig. 1. Exponential approximation of fatigue crack.

$T_{d}=\left(\log a_{d}-\log \alpha\right) / Q=C_{d} / Q$

$T_{c}=\left(\log a_{c}-\log \alpha\right) / Q=C_{c} / Q$

Let us denote $X=\log Q$ and $Y=\log C_{c}$, where $C_{c}=\log a_{c}-\log \alpha$. From the analysis of the fatigue test data it can be assumed that $\log T_{c}=\log C_{c}-\log Q$ is distributed normally. It results from the additive property of the normal distribution that can take place if either both $\log C_{c}$ and $\log Q$ are normally distributed or if one of these components is normally distributed, while the other is constant. Contrary to [1], in this paper we consider the first case: vector $(X, Y)=\left(\log (Q), \log \left(C_{c}\right)\right)$ has twodimensional normal distribution with vectorparameter $\theta=\left(\mu_{X}, \mu_{Y}, \sigma_{X}, \sigma_{Y}, r\right)$. It is worth noting that for the case when $a_{c}$ and $a_{d}$ are constants, cdf of $C_{d}$ is completely defined by the distribution of $C_{c}$ because $C_{d}=C_{c}-\delta$, where $\delta=\log \left(a_{c} / a_{d}\right)$. When $\theta$ is known, there are two decisions $d_{0}$ and $d_{1}$ : the aircraft is good enough and the operation of this aircraft type can be allowed $\left(d_{0}\right)$ or the redesign of aircraft should be carried out $\left(d_{1}\right)$. In case of the first decision, vector $\vec{t}=\left(t_{1}, \ldots, t_{n}\right)$, where $t_{i}$ is the time moment of $i$-th inspection, should also be defined. If $\theta$ is known the different rules can be offered for the choice of structure of vector $\vec{t}: 1)$ every interval between inspections is equal to $\left.t_{S L} /(n+1), 2\right)$ probability of failure in every interval 
is equal to $P\left(T_{C}<t_{S L}\right) /(n+1) \ldots$ In this paper we suppose that (just as in the above-mentioned examples) vector $\vec{t}$ is defined by means of fixed $t_{S L}$ and choice of $n$.

To substantiate the choice of inspection number, we should know FFP of AC and FFR and gain (GL) of AL as functions of $n$. For this purpose, the process of operation of AC can be viewed as absorbing MC with $(n+4)$ states. States $E_{1}, E_{2}, \ldots, E_{n+1}$ correspond to $\mathrm{AC}$ operation in time intervals $\left[t_{0}, t_{1}\right),\left[t_{1}, t_{2}\right), \ldots,\left[t_{n}, t_{S L}\right)$, and states $E_{n+2}, E_{n+3}$, and $E_{n+4}$ are absorbing states: AC is discarded from service when SL is reached or there is a fatigue failure (FF), or fatigue crack detection (CD) takes place (see Fig. 2).

\begin{tabular}{|c|c|c|c|c|c|c|c|c|c|c|}
\hline & $\mathrm{E}_{1}$ & $\mathrm{E}_{2}$ & $\mathrm{E}_{3}$ & $\ldots$ & $\mathrm{E}_{\mathrm{n}-1}$ & $\mathbf{E}_{\mathrm{u}}$ & $\mathbf{E}_{\mathbf{n}+1}$ & $\underbrace{\mathbf{E}_{\text {(SL) }}}_{\text {(St2) }}$ & $\begin{array}{l}\mathbf{E}_{n+3} \\
(\mathrm{FF})\end{array}$ & $\begin{array}{l}\mathrm{E}_{\mathrm{n}+4} \\
\text { (CD) }\end{array}$ \\
\hline $\mathrm{E}_{1}$ & 0 & $\mathrm{u}_{1}$ & 0 & $\ldots$ & 0 & 0 & 0 & 0 & $q_{1}$ & $v_{1}$ \\
\hline $\mathbf{E}_{2}$ & 0 & 0 & $\mathrm{u}_{2}$ & $\ldots$ & 0 & 0 & 0 & 0 & $q_{2}$ & $v_{2}$ \\
\hline $\mathrm{E}_{3}$ & 0 & 0 & 0 & $\ldots$ & 0 & 0 & 0 & 0 & $q_{3}$ & $v_{3}$ \\
\hline$\ldots$ & & & $\ldots$ & $\ldots$ & & $\ldots$ & $\ldots$ & $\ldots$ & $\ldots$ & $\ldots$ \\
\hline$E_{n-1}$ & 0 & 0 & 0 & $\ldots$ & 0 & $\mathrm{u}_{\mathrm{n}-1}$ & 0 & 0 & $q_{n-1}$ & $v_{n-1}$ \\
\hline $\mathbf{E}_{\mathrm{n}}$ & 0 & 0 & 0 & $\ldots$ & 0 & 0 & $\mathrm{u}_{\mathrm{n}}$ & 0 & $q_{\mathrm{n}}$ & $v_{n}$ \\
\hline$E_{n+1}$ & 0 & 0 & 0 & $\ldots$ & 0 & 0 & 0 & $u_{n+1}$ & $q_{n+1}$ & $v_{n+1}$ \\
\hline $\begin{array}{l}\mathrm{E}_{\mathrm{n}+2} \\
(\mathbf{S L L})\end{array}$ & 0 & 0 & 0 & & 0 & 0 & 0 & 1 & 0 & 0 \\
\hline $\begin{array}{l}\mathrm{E}_{n+3} \\
(\mathrm{Fr})\end{array}$ & 0 & 0 & 0 & $\cdots$ & 0 & 0 & 0 & 0 & 1 & 0 \\
\hline $\begin{array}{l}E_{n+4} \\
\text { (CD) }\end{array}$ & 0 & 0 & 0 & $\ldots$ & 0 & 0 & 0 & 0 & 0 & 1 \\
\hline
\end{tabular}

Fig. 2. Transition probability matrix $P_{A C}$.

In the transition probability matrix, $P_{A C}$, for corresponding process of AC operation let the probability of crack detection during the inspection number $i$ be denoted as $v_{i}(6)$; probability of failure in service time interval $t \in\left(t_{i-1}, t_{i}\right]$ be denoted as $q_{i}$ (5) and probability of successful transition to the next state as $u_{i}(6)$. In our model we also assume that an aircraft is discarded from service at $t_{S L}$ even if there are no cracks discovered by inspection at time moment $t_{S L}$. This inspection at the end of ( $n+1)$-th interval (in state $E_{n+1}$ ) does not change reliability but it is carried out in order to know the state of an aircraft (whether there is a fatigue crack or there is no fatigue crack). It can be shown in equations (4-6) where $\mathrm{a}_{\mathrm{i}}$, $\mathrm{g}_{\mathrm{ai}}, \mathrm{b}_{\mathrm{i}}, \mathrm{g}_{\mathrm{bi}}, \mu_{X / y}, \sigma_{X / y}$ is defined in equations (7-12)

$u_{i}=P\left(T_{d}>t_{i} \mid T_{d}>t_{i-1}\right)=$

$=P\left(Q<C_{d} / t_{i}\right) / P\left(Q<C_{d} / t_{i-1}\right)=a_{i} / a_{i-1}$,
$q_{i}=P\left(t_{i-1}<T_{d}<T_{c}<t_{i} \mid T d>t_{i-1}\right)=$

$=\left\{\begin{array}{l}0, \quad \text { if } t_{i-1} C_{c} / C_{d}>t_{i}, \\ b_{i} / a_{i-1}, \quad \text { if } t_{i-1} C_{c} / C_{d} \leq t_{i},\end{array} \quad i=1, \ldots, n+1\right.$,

$v_{i}=1-u_{i}-q_{i}$,

where

$a_{i}=P\left(Q<C_{d} / t_{i}\right)=\int_{\ln \delta}^{+\infty}\left(g_{a i}(y)\right) d \Phi\left(\frac{y-\mu_{Y}}{\sigma_{Y}}\right)$,

$g_{a i}=P\left(Q<C_{d} / t_{i}\right)=$

$=\Phi\left(\frac{\left(\log \left(e^{y}-\delta\right)-\log t_{i}\right)-\mu_{X / y}}{\sigma_{X / y}}\right)$,

$b_{i}=P\left(C_{c} / t_{i}<Q<C_{d} / t_{i-1}\right)$,

$=P\left(\log C_{c}-\log t_{i} \leq \log Q<\log \left(C_{c}-\delta\right)-\log t_{i-1}\right)$,

$=\int_{\ln \delta}^{+\infty}\left(g_{b i}(y)\right) d \Phi\left(\frac{y-\mu_{Y}}{\sigma_{Y}}\right)$,

$g_{b i}(y)=\max \left(\begin{array}{l}0, \Phi\left(\frac{\left(\log \left(e^{y}-\delta\right)-\log t_{i-1}\right)-\mu_{X / y}}{\sigma_{X / y}}\right) \\ -\Phi\left(\frac{\left(y-\log t_{i}\right)-\mu_{X / y}}{\sigma_{X / y}}\right)\end{array}\right)$,

$\mu_{X / y}=\mu_{X}+r \frac{\sigma_{X}}{\sigma_{Y}}\left(y-\mu_{Y}\right)$,

$\sigma_{X / y}=\sigma_{X} \sqrt{1-r^{2}}$

These probabilities can also be calculated using the Monte Carlo method (13). Equation (14) can be used for modelling r.v with some coefficient of correlation $r$ where r.v. $\eta_{1}$ and $\eta_{2}$ have the standard normal distribution.

$Y=\log C_{c} \sim N\left(\mu_{Y}, \sigma_{Y}^{2}\right), X=\log Q \sim N\left(\mu_{X}, \sigma_{X}^{2}\right)$

$Y=\eta_{1} \sigma_{Y}+\mu_{Y}, X=\eta_{1} \sigma_{X} r+\eta_{2} \sigma_{X} \sqrt{1-r^{2}}+\mu_{X}$

Let us recall that in the matrix, $P_{A C}$, there are three units in three last lines in a diagonal matrix because states $E_{n+2}$, $E_{n+3}$, and $E_{n+4}$ are absorbing states: AC is discarded from 
service when SL is reached or there is a fatigue failure (FF), or fatigue crack detection $(\mathrm{CD})$ takes place.

In the corresponding matrix for operation process of AL, states $E_{n+2}, E_{n+3}$ and $E_{n+4}$ are not absorbing ones and correspond to return of $\mathrm{MC}$ to state $E_{1}$ (AL operation returns to the first interval). The other lines of $P_{A C}$ and $P_{A L}$ are the same.

For SMPW version of problem, by using $P_{A L}$ we can obtain the airline gain (15), where $\pi=\left(\pi_{1}, \ldots, \pi_{n+4}\right)$ is the vector of stationary probabilities, which is defined by (16)

$g(n)=\sum_{i=1}^{n+4} \pi_{i} g_{i}(n)$

$\pi P=\pi, \quad \sum_{i=1}^{n+4} \pi_{i}=1$

$g_{i}(n)=\left\{\begin{array}{l}a_{i} \cdot u_{i}+b_{i} \cdot q_{i}+c_{i} \cdot v_{i}, \quad i=1, \ldots n+1, \\ d_{i}, \quad i=n+2, \ldots, n+4,\end{array}\right.$

AL operation rewards are defined in (17), where $a_{i}$ is the reward related to a successful transition from one operation interval to the next one and the cost of one inspection; $b_{i}, c_{i}$ and $d_{i}$ are related to the transition to states $E_{n+3}$ (FF), $E_{n+4}$ (CD) and $E_{1}$. Let us note that if $a=b=c=1, d=0$ time transition to state $E_{l}$ equals zero, then $\pi_{t j}=\pi_{j} g_{j}(n) / g(n)$ defines the time, which is spent by SMP in state $E_{j} j=1, \ldots ., n+1, L_{j} g(n) / \pi_{j}$ defines the mean return time for state $E_{j}$.

Specifically, $L_{n+3}$ is the mean time between FF; so $\lambda_{F}=1 / L_{n+3}$ is FFR. It is also worth mentioning that the same value can be calculated in another way. This value is equal to the ratio of aircraft failure probability, $p_{F}$, to the mean life of a new aircraft, $L_{1}=g(n) / \pi_{1}$ (the mean time of renewal of $\mathrm{AC}$ (renewal operation of AL in the first interval)).

There are two versions of reliability requirements: A) limitation of FFR of AL; and B) limitation of FFP of AC. We will thoroughly consider case A. If $\theta$ is known, we calculate the gain as a function of $n, g(n, \theta)$, and choose number $n_{g}$ corresponding to the maximum of gain. Then we calculate FFR as a function of $n, \lambda_{F}(n, \theta)$, and choose $n_{\lambda}$ in such a way that for all $n \geq n_{\lambda}$ function $\lambda_{F}(n, \theta)$ will be equal to or less than some value $\lambda_{F D}$ (the "designed" FFR) (18). Finally, we choose inspection number n (19).

$$
\begin{aligned}
& n_{\lambda}\left(\lambda_{F D}, \theta\right)= \\
& =\min \left\{n: \lambda_{F}(n, \theta) \leq \lambda_{F D}, \text { for all } n \geq n_{\lambda}\left(\lambda_{F D}, \theta\right)\right\} \\
& n=n_{g \lambda}\left(\lambda_{F D}, \theta\right)=\max \left(n_{g}(\theta), n_{\lambda}\left(\lambda_{F D}, \theta\right)\right)
\end{aligned}
$$

However, we do not know $\theta$ and we can get only some estimate of this parameter, $\hat{\theta}$. Then, first of all, we should define some part of parameter space $\Theta_{0}$ in such a way that if $\hat{\theta} \notin \Theta_{0}$ then redesign of AC should be carried out.

If instead of $n_{g \lambda}\left(\lambda_{F D}, \theta\right)$ we use $\hat{n}_{g \lambda}=n_{g \lambda}\left(\lambda_{F D}, \hat{\theta}\right)$ then real intensity FFR will be a function of random variable, $\lambda_{F}\left(\hat{n}_{g \lambda}, \theta\right)$. Let us define $\lambda_{F}\left(\hat{\theta}, \lambda_{F D}, \Theta_{0}\right)=\lambda_{F}\left(\hat{n}_{g \lambda}, \theta\right)$ if $\hat{\theta} \in \Theta_{0}$ and $\lambda_{F}\left(\hat{\theta}, \lambda_{F D}, \Theta_{0}\right)=0$ if $\hat{\theta} \notin \Theta_{0}$ (service of this type of AC is not allowed). The corresponding expected value of FFR as a function of $\theta$ has its maximum because in case of "bad $\hat{\theta}$ " we redesign an airframe, but in case of "very good $\hat{\theta}$ " we do not need any inspection.

Let us denote by $\lambda_{F D}^{*}\left(\Theta_{0}\right)$ the solution to (20) (if there is the solution to this equation for specific $\left.\Theta_{0}\right)$, where $w_{\lambda}(21)$ is 'required FFR' defined by specific aviation regulations.

$\sup _{\theta} w_{\lambda}\left(\theta, \lambda_{F D}, \Theta_{0}\right)=\lambda^{*}$

$w_{\lambda}\left(\theta, \lambda_{F D}, \Theta_{0}\right)=E\left\{\lambda_{F}\left(\hat{\theta}, \lambda_{F D}, \Theta_{0}\right)\right\}, \lambda^{*}$

$w_{\lambda}\left(\theta, \lambda_{F D}, \Theta_{0}\right)=$

$=\left\{\int_{\Theta_{0}} \lambda_{F}\left(n_{g \lambda}\left(\lambda_{F D}, x\right), \theta\right) *\right.$

$\left.* d F_{\hat{\theta}}\left(x \mid \hat{\theta} \in \Theta_{0}\right)\right\} P\left(\hat{\theta} \in \Theta_{0}\right)$.

$w_{p}\left(\theta, p_{F D}, \Theta_{0}\right)=$

$=\sum_{i=1}^{n+1} P\left(t_{i-1}(\hat{\theta}) \leq T_{d}<T_{c}<t_{i}(\hat{\theta}), \hat{\theta} \in \Theta_{0}\right)$,

$n_{p}\left(p_{F D}, \theta\right)=$

$=\min \left\{n: p_{F}(n, \theta) \leq p_{F D}\right.$, for all $\left.n \geq n_{p}\left(n_{F D}, \theta\right)\right\}$,

If after the approval test we see that $\hat{\theta} \in \Theta_{0}$ then required inspection number $n=n_{g \lambda}\left(\lambda_{F D}^{*}, \hat{\theta}\right)$. In a similar way the choice of $n$ can be made for case B. Instead of (22) where $F_{\hat{\theta}}($.$) is cdf of \hat{\theta}$, the following p-set function [1] should be used (23), where $t_{0}=0, t_{n+1}=t_{S L}, t_{i}(\theta), i=1, \ldots, \hat{n}_{g p}$,

$\hat{n}_{g p}=\max \left(n_{g}, n_{p}\left(p_{F D}, \hat{\theta}\right)\right), p_{F D}$ is "designed" as allowed FFP of AC, which is used for the choice of $n_{p}$ (24), function $p_{F}(n, \theta)$ defines FFP of AC for specific $n$ and $\theta$. 
TABLE I

FATIGUE CRACK PARAMETERS

\begin{tabular}{|l|l|l|l|l|l|}
\hline No. & Crack \# & $\operatorname{Ln}(\mathrm{a} 0)$ & $\mathrm{Q}$ & $\mathrm{X}=\operatorname{Ln}(\mathrm{Q})$ & $\mathrm{Y}=\operatorname{lnCc}$ \\
\hline 1 & 75 & -1.2513 & $1.86 \mathrm{E}-04$ & -8.58976 & 1.905519 \\
\hline 2 & 92 & -1.8768 & $1.95 \mathrm{E}-04$ & -8.54251 & 1.994482 \\
\hline 3 & 93 & -1.2445 & $1.61 \mathrm{E}-04$ & -8.73411 & 1.904507 \\
\hline 4 & 116 & -1.697 & $2.20 \mathrm{E}-04$ & -8.42188 & 1.96971 \\
\hline 5 & 112 & -1.5102 & $2.07 \mathrm{E}-04$ & -8.48279 & 1.943306 \\
\hline 7 & 77 & -2.5329 & $2.28 \mathrm{E}-04$ & -8.38616 & 2.080003 \\
\hline 8 & 78 & -0.6479 & $1.54 \mathrm{E}-04$ & -8.77856 & 1.81148 \\
\hline 10 & 129 & -1.4226 & $1.57 \mathrm{E}-04$ & -8.75926 & 1.93068 \\
\hline & Average & -1.5229 & 0.000189 & -8.5868804 & 1.942461 \\
\hline & StdDev & 0.5480844 & $2.9 \mathrm{E}-05$ & 0.1551287 & 0.077889 \\
\hline & CORREL $\mathrm{r}$ & & & 0.796 \\
\hline
\end{tabular}

\section{NUMERICAL EXAMPLE}

In Table 2.1 of [1] some a priori information about a fatigue crack growth function is provided. Using this information for $a_{c}=237.8 \mathrm{~mm}$ and $a_{d}=20 \mathrm{~mm}$ (these value have already been used in [1]) we get the following estimate of parameter

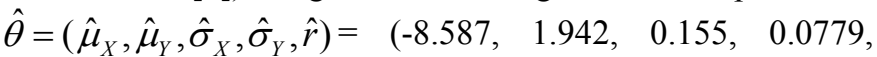
0.796) (see Table I). It is supposed that all inspection intervals are equal. The following definition of components of $\mathrm{AL}$ income is used: for all $i=1, \ldots, n+1$ $a_{i}=a(n)=a_{0}(n)+d_{\text {insp }} t_{S L}, \quad$ where $a_{0}(n)=a_{01} t_{S L} /(n+1)$ is the reward related to a successful transition from one operation interval to the next one; $a_{01}$ defines the reward of operation in one time unit (one hour or one flight); $d_{\text {insp }} t_{S L}$ is the cost of one inspection (a negative value), which is supposed to be proportional to $t_{S L} ; b_{i}=b_{01} t_{S L}$ is related to FF (a negative value), $c_{i}=c_{01} a_{0}(n)$ is the reward related to transitions from any state $E_{1}, \ldots, E_{n+1}$ to state $E_{n+4}$ (it is supposed to be proportional to $a_{0}$ because it is part of $a_{0}$ ); $d_{i}=d_{01} t_{S L}$ is a negative reward, the absolute value of which is the cost of new aircraft acquisition after events SL, FF or CD and the transition to $E_{1}$. In the numerical example we have used the following values (see Table II).

TABLE II

ECONOMIC PARAMETERS

\begin{tabular}{|l|l|l|}
\hline Symbol & Event & Values \\
\hline$b_{01}$ & Transition to state $E_{n+3}(\mathrm{FF})$ & -3 \\
\hline$d_{\text {insp }}$ & Inspection cost & -0.05 \\
\hline$a_{01}$ & $\begin{array}{l}\text { Reward related to a successful transition } \\
\text { from one operation interval to the next one }\end{array}$ & 1 \\
\hline$c_{01}$ & Transition to state $E_{n+4}(\mathrm{CD})$ & 0.05 \\
\hline$d_{01}$ & Transition to state $E_{1}$ & -0.3 \\
\hline
\end{tabular}

Set $\Theta_{0}$ is defined in the following way: the redesign of the AC type should be carried out if an estimate of mean AC life is small $\left(T_{c}<t_{S L}\right)$ or a speed of fatigue crack growth is large $\left(\log \mathrm{Q}>\hat{\mu}_{X}+\sigma_{X}\right)$. For $\lambda_{F D}=0.0000001$ in Fig. 3a the results of calculation of $w_{\lambda}\left(\theta, \lambda_{F D}, \Theta_{0}\right)$ and corresponding Fig. $3 \mathrm{~b}$ (25), where $\quad t_{0}=0, t_{n+1}=t_{S L}, t_{i}(\theta), i=1, \ldots, \hat{n}_{g \lambda}$, $\hat{n}_{g \lambda}=n_{g \lambda}\left(\lambda_{F D}, \hat{\theta}\right)$, as a function of $\mu_{X}$ for $\left(\mu_{Y 1}, \ldots, \mu_{Y 5}\right)=$ $(1.55,1.75,1.94,2.14,2.33)$ in the vicinity of its maximum are shown.

$w_{p \lambda}\left(\theta, \lambda_{F D}, \Theta_{0}\right)=$
$=\sum_{i=1}^{n+1} P\left(t_{i-1}(\hat{\theta}) \leq T_{d}<T_{c}<t_{i}(\hat{\theta}), \hat{\theta} \in \Theta_{0}\right)$

It is supposed that vector $\left(\sigma_{X}, \sigma_{Y}, r\right)$ is the same for different vectors $\left(\mu_{X}, \mu_{Y}\right)$ and it is equal to the test estimate $(0.155128668,0.0778895,0.796437)$. (Let us recall that $\left(\mu_{Y}-\mu_{X}\right)$ is equal to $\left.E\left(\log \left(T_{C}\right)\right)\right)$.

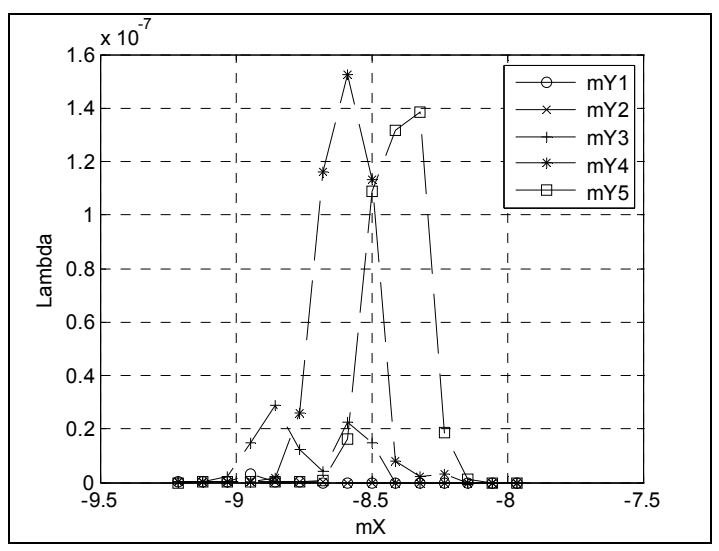

Fig. 3a. $w_{\lambda}\left(\theta, \lambda_{F D}, \Theta_{0}\right)$ as a function of $\mu_{X}$.

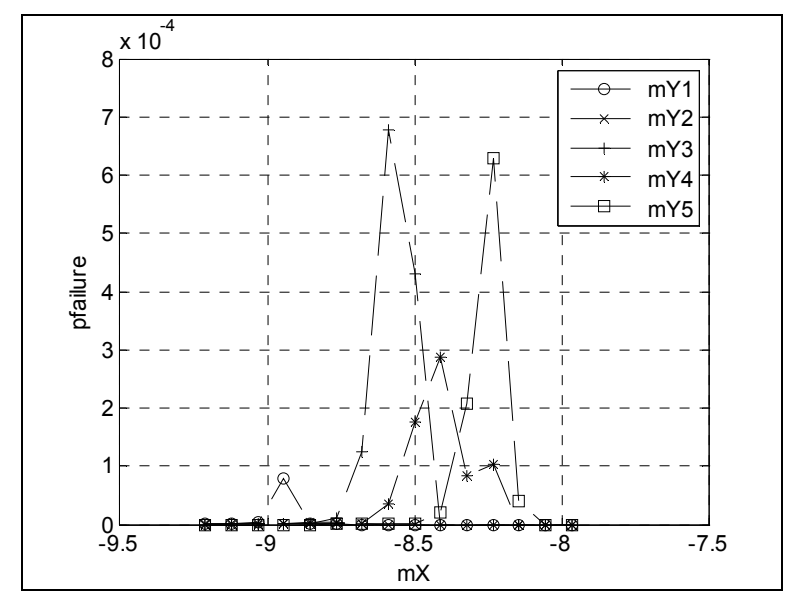

Fig. 3b. $w_{\lambda}\left(\theta, \lambda_{F D}, \Theta_{0}\right)$ as a function of $\mu_{X}$. 
Maximum value of $w_{\lambda}\left(\theta, \lambda_{F D}, \Theta_{0}\right)$ is equal to $1.523 * 10^{-7}$. Maximum value of $w_{p \lambda}\left(\theta, \lambda_{F D}, \Theta_{0}\right)$ is equal to 0.000677 . Let us suppose that these values satisfy the requirements: required FFR of $\mathrm{AL}$ and required FFP of $\mathrm{AC}$. Now let us suppose that in a real test we have got $\hat{\mu}_{X}=-8.5885$, $\hat{\mu}_{Y}=1.942460769$ (see Fig. 4). (These values have already been considered in [1]). After calculating FFR and airline gain (see Fig. 5), for these specific parameters we find a required number of inspections: $n=\max \left(n_{g}, n_{\lambda}\right)=\max (3,4)=4$. It appears that the influence of scatter of EIFS, $\alpha$, is very significant. After similar calculations of $w_{\lambda}\left(\theta, \lambda_{F D}, \Theta_{0}\right)$ as a function of $\mu_{X}$ for $\sigma_{Y}=0.00001, r=0$ we get its maximum value equal to $1.87 * 10^{-8}$. It is nearly 10 times lower than in the previous case (when $\sigma_{Y}=0.0778895$ ). Therefore, it is very important to take into account the scatter of EIFS, $\alpha$.

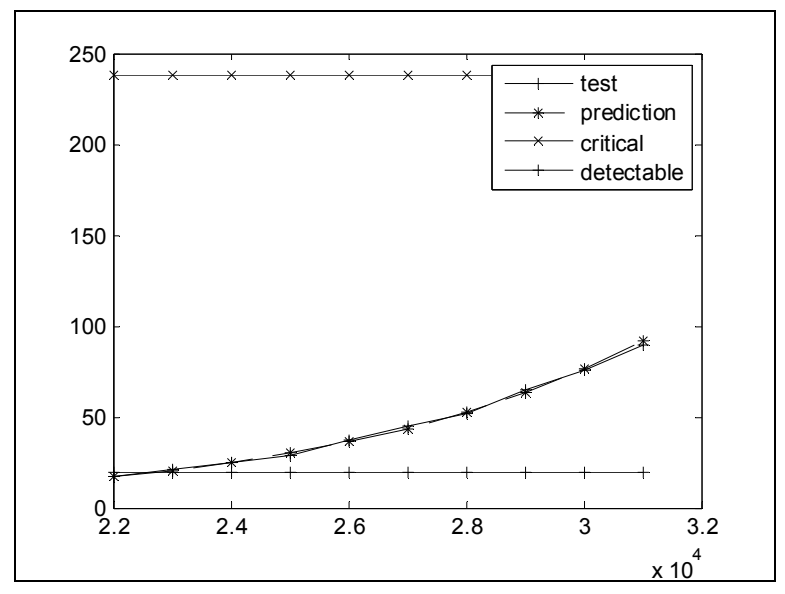

Fig. 4. Example of fatigue crack size as a function of flight number.

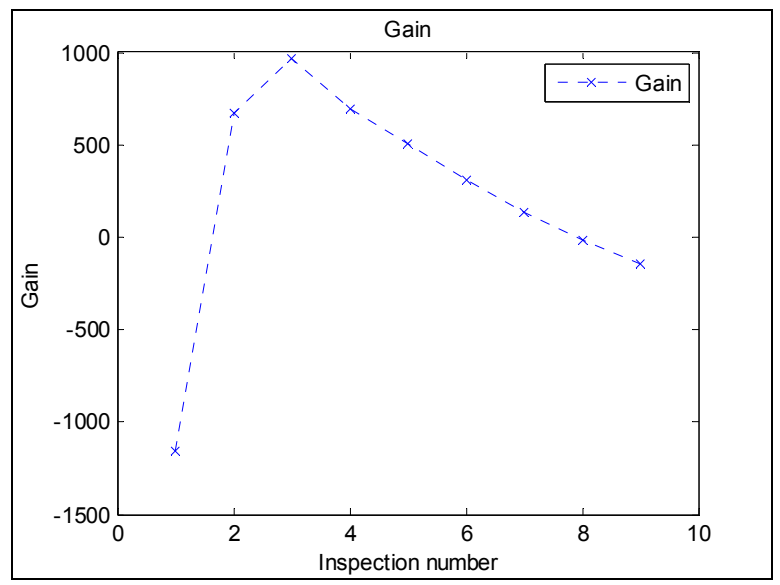

Fig. 5a. Airline gain as a function of inspection number for specific $\hat{\mu}_{X}=-$ $8.5885, \hat{\mu}_{Y}=1.942460769$.

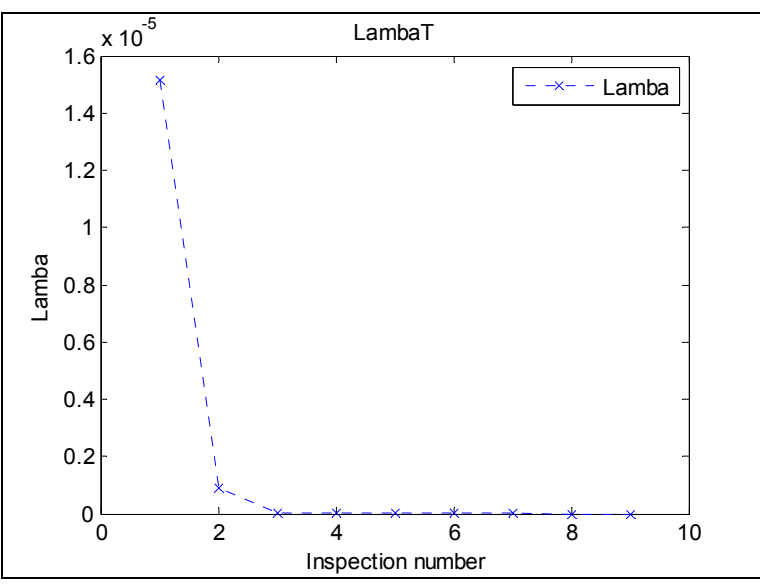

Fig. 5b. FFR as a function of inspection number for specific $\hat{\mu}_{X}=-8.5885$, $\hat{\mu}_{Y}=1.942460769$.

\section{CONCLUSIONS}

Here is shown as the full-scale fatigue approval test of an airframe can be used for aircraft inspection program develop, based not only on limitation of fatigue failure rate, but extends to the economic analysis of results the best likelihood is achieved using minmax method.

\section{ACKNOWLEDGEMENTS}

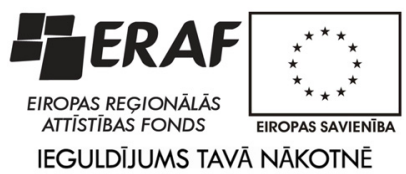

The research has been supported by the European Regional Development Fund within the framework of project "Development of an Unmanned Aircraft System and Creation of the Industrial Prototypes of Unmanned Aerial Vehicles for Performing the Tasks of Latvian National Economy" No.2010/0256/2DP/2.1.1.1.0/10/APIA/VIAA/070

\section{REFERENCES}

[1] Y. Paramonov, A. Kuznetsov and M. Kleinhofs, Reliabilty of fatigueprone airframes and composite materials, Riga, RTU Publishing house, 2011.

[2] M. Hauka and Y. Paramonovs, Inspection Program Development Using Minimax Method: $11^{\text {th }}$ International Conference "Reliability and Statistics in Transportation and Communication", pp. 105-114, October 19-22, 2011 Riga, Latvia.

[3] M. Hauka and Y. Paramonovs, Inspection Program for the Case of Two Random Parameters of Fatigue Crack: $12^{\text {th }}$ International Conference "Reliability and Statistics in Transportation and Communication", pp. 78-84, October 17-20, Riga, Latvia.

[4] J. R. Mohanty, B. B. Vermaa and P. K. Ray, "Prediction of fatigue crack growth and residual life using an exponential model: Part I (constant amplitude loading)," International Journal of Fatigue, vol.31, issue 3, pp. 418-424, March 2009. http://dx.doi.org/10.1016/j.ijfatigue.2008.07.015 
[5] J. R. Mohanty, B. B. Verma and P. K. Ray, "Prediction of fatigue crack growth and residual life using an exponential model: Part II (mode-I overload induced retardation)," International Journal of Fatigue, vol.31, issue 3, pp. 425-432, March 2009.

http://dx.doi.org/10.1016/j.ijfatigue.2008.07.018

[6] K. Sobczyk, "Modelling of random fatigue crack growth," Journal of Engineering Fracture Mechanics, vol. 24, issue 4, pp. 609-623, 1986. http://dx.doi.org/10.1016/0013-7944(86)90234-1

[7] R. De Silvar, Exponential model of fatigue crack: In Proceeding of the International workshop "Aviation Reliability AvR 2000, pp. 45-48, May 9-10, 2000, Riga, RAU, 2000.

[8] B. F. Jr. Spencer and J. Tang, "Markov process model for fatigue crack growth," Journal of Engineering Mechanics, vol. 114, issue 12, pp. 2134-2157, 1988

[9] J. C. Newman, J. W Shaw, B. S. Annigeri and B. M. Ziegler, "Fatigue and Crack Growth in 7050-T7451 Aluminium Alloy Under Constant and Variable-Amplitude Loading," Journal of Engineering for Gas Turbines Power, vol. 135(2), January 2013. http://dx.doi.org/10.1115/1.4007755

[10] R. A. Howard, Dynamics programming and Markov Processes. New York-London: Wiley, 1960.

[11] J. R. Norris, Markov Chains. Cambridge: Cambridge University Press, 1997. http://dx.doi.org/10.1017/CBO9780511810633

[12] F. Kachapova, "Representing Markov chains with transition diagrams," Journal of Mathematics \& Statistics. vol. 9, issue 3, pp. 149-154, 2013. http://dx.doi.org/10.3844/jmssp.2013.149.154

[13] R. B. Thompson, "A Unified Approach to the Model-Assisted Determination of Probability of Detection," Materials Evaluation, vol. 66, issue 6, pp. 667-673, June 2008.

[14] J. S. Knopp et al. "Investigation of a Model-Assisted Approach to Probability of Detection Evaluation," In: D. O. Thompson and d. E. Chimenti (eds.), Review of Progress in Quantitative Nondestructive Evaluation, vol. 26, pp. 1775-1782, New York, USA: American Institute of Physics, 2007.

[15] C. A. Harding, G. R. Hugo and S. J. Bowles, "Application of ModelAssisted POD Using a Transfer Function Approach,” In: In: D. O Thompson and d. E. Chimenti (eds.), Review of Progress in Quantitative Nondestructive Evaluation, vol. 28, pp. 1792-1799, New York, USA American Institute of Physics, 2009.

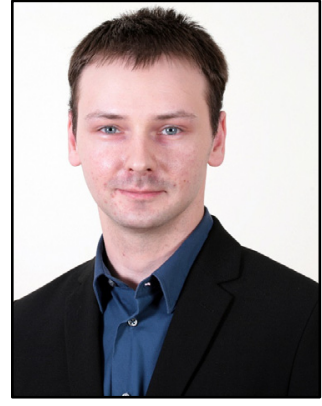

Maris Hauka in 2009 graduated from Riga Aviation University (former Riga Civil Aviation Engineering Institute), Faculty of Transport and Mechanical Engineering and received a degree of Master of Engineering Science.

Present position: Lecturer and $\mathrm{PhD}$ candidate at the Institute of Aeronautics, Riga Technical University.

His fields of research: reliability of technical systems.

Institute of Aeronautics, Faculty of Transport and Mechanical Engineering, Riga Technical University, Lomonosova 1A, k-1, Riga, LV-1019, Latvia.

Phone: +37167089990

E-mail: Maris.Hauka@rtu.lv

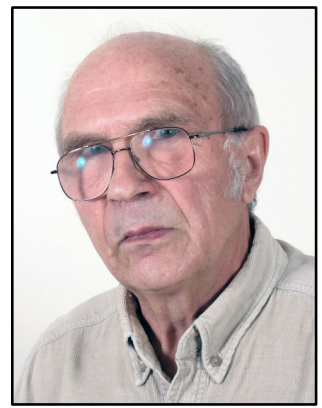

Jurijs Paramonovs. in 1993 was awarded a degree of Hab.Dr.eng by Riga Aviation University and the Latvian Academy of Sciences. In 1974 he was awarded a Doctoral Degree in Technical Cybernetics by the Latvian Academy of Sciences. In 1965 he received a degree of Doctor of Engineering Science from Riga Civil Aviation Engineering Institute.

Present Position: Professor of Aircraft Theory and Structure Department at the Aeronautical Institute of Riga Technical University.

Member of professional societies: American Statistical Association, Research Board of Advisors of the American Biographical Institute. He has participated in 17 international conferences and has written 145 publications, including 9 monographs and textbooks.

His fields of research: reliability of technical systems; mathematical statistics; loads, structure and strength analysis of transport vehicle; information technology application (development of automated control systems).

Institute of Aeronautics, Faculty of Transport and Mechanical Engineering, Riga Technical University, Lomonosova 1A, k-1, Riga, LV-1019, Latvia. Phone: +37167089990

E-mail: Jurijs.Paramonovs@rtu.lv 